\title{
Parámetros Electrocardiográficos en Terneros Holstein Criados en la Altura y a Nivel del Mar
}

\author{
Electrocardiographic Parameters in Holstein Calves Reared at High \\ Altitude And at Sea LeVel
}

\author{
Christian Begazo C. ${ }^{1,3}$, Harnold Portocarrero P. ${ }^{1}$, Roberto Dávila F. ${ }^{2}$
}

\section{Resumen}

El propósito del estudio fue comparar los parámetros electrocardiográficos de terneros Holstein, clínicamente sanos, criados en la altura (3827 msnm) y a nivel del mar (175 msnm). Se evaluó la frecuencia, ritmo, tamaño y morfología de las ondas, segmentos e intervalos y el eje cardíaco. Los exámenes se realizaron en posición erguida, a una amplitud de $1 \mathrm{mV} / \mathrm{cm}$ y a velocidad de $25 \mathrm{~mm} / \mathrm{seg}$, utilizándose las derivaciones bipolares (I, II, III) y unipolares (avL, avR y avF). Los resultados reflejaron que la frecuencia cardíaca en la altura y a nivel del mar fue de $87.33 \pm 9.77$ y de $127.40 \pm 7.52$ latidos por minuto, respectivamente. El ritmo cardíaco en la altura y a nivel del mar tuvo como característica un ritmo regular sinusal normal. El eje cardíaco en la altura fue de $-120.1 \pm 7.30^{\circ} \mathrm{y}$ a nivel del mar de $63.7 \pm 3.02^{\circ}$. Se concluye que existen diferencias estadísticas en frecuencia cardíaca, tamaño y morfología de las ondas, intervalos, segmentos y el eje cardíaco en los terneros de la altura y a nivel del mar $(\mathrm{p}<0.05)$, producto de la adaptación fisiológica a niveles altitudinales mayores.

Palabras clave: parámetros electrocardiográficos, eje cardíaco, terneros

\section{Abstract}

The aim of the study was to compare the electrocardiographic parameters of clinically healthy Holstein calves raised in the high altitude $(3827 \mathrm{~m})$ and at sea level $(175 \mathrm{~m})$. Parameters under evaluation were rate, rhythm, size and morphology of the waves, segments and intervals and cardiac axis. The tests were performed in an upright position at an amplitude of $1 \mathrm{mV} / \mathrm{cm}$ and speed of $25 \mathrm{~mm} / \mathrm{sec}$, using the bipolar (I, II, III) and unipolar (AVL, AVR, and aVF) leads. The results showed that heart rate was $87.33 \pm 9.77$

\footnotetext{
${ }^{1}$ Laboratorio de Patología Clínica, Universidad Nacional del Altiplano, Puno, Perú

${ }^{2}$ Clínica de Animales Menores, Facultad de Medicina Veterinaria, Universidad Nacional Mayor de San Marcos, Lima, Perú

${ }^{3}$ E-mail: Sbc27@hotmail.com
}

Recibido: 3 de diciembre de 2015

Aceptado para publicación: 28 de noviembre de 2016 
and $127.40 \pm 7.52$ at high altitude and at sea level respectively. The heart rate at both locations were considered as regular normal sinus rhythm. The cardiac axis at high altitude was $-120.1 \pm 7.30^{\circ}$ and at sea level was $63.7 \pm 3.02^{\circ}$. It is concluded that there were statistical differences $(\mathrm{p}<0.05)$ in heart rate, size and morphology of the waves, intervals, segments and cardiac axis due to the physiological adaptation to the high altitude.

Key words: electrocardiographic parameters, heart axis, calves

\section{INTRODUCCIÓN}

En las regiones andinas se encuentra una gran parte de la ganadería bovina periurbana, la cual viene realizando un proceso de intensificación para la producción de leche (FAO, 2008). En este ambiente, el ganado Holstein enfrenta dificultades de adaptación a la altitud, principalmente problemas de origen cardíaco (Ayón y Cueva, 1998).

Los animales en general, viven en un estado de cercana interacción entre la complejidad de los procesos físicos y químicos de su propio cuerpo y el entorno que los rodea, de tal forma que en el proceso de adaptación, tanto el clima como la altitud son factores que pueden afectar significativamente el desempeño económico del mismo (Fregin, 1985; Claxton, 1988). Estas características determinan el comportamiento fisiológico de las especies animales que habitan en estas zonas, aunque estas diferencias no siempre están claramente establecidas (Ayón y Cueva, 1998).

La compleja interacción animal-ambiente en elevados niveles altitudinales determina la presencia de diversas alteraciones, entre ellas las cardiovasculares, que en bovinos generalmente provocan gran morbilidad y mortalidad (Ayón y Cueva, 1998). Es así que el estudio de los factores determinantes permite conocer las constantes y parámetros fisiológicos responsables de la morbimortalidad y menor rendimiento productivo de las especies en la altitud (Arango et al., 1992).
El ganado bovino, al igual que otras especies, puede estar adaptado a las condiciones medioambientales y de manejo de su entorno; sin embargo, pueden pasar por situaciones de estrés debido a oscilaciones en la temperatura medioambiental (West, 2003). Las zonas de altura se caracterizan por la baja presión de oxígeno y el frío, los cuales afectan a la mayoría de las especies animales, siendo el bovino una de las más susceptibles (Ayón y Cueva, 1998).

Uno de los métodos más empleados para el diagnóstico de disfunciones cardiovasculares es el electrocardiograma, que se constituye en una herramienta básica aunque su uso más frecuente es en pequeños animales (Tilley y Lee, 2000). Su uso en animales mayores como el bovino y en la raza Holstein es aún incipiente, siendo una de las razones el escaso conocimiento de las variables fisiológicas normales en diferentes condiciones de altitud. Es así que el presente estudio tuvo como objetivo caracterizar los parámetros electrocardiográficos del bovino Holstein en la altura y a nivel del mar.

\section{Materiales y Métodos}

Se evaluaron 16 terneros Holstein, clínicamente sanos y con edades entre 1 y 6 meses. Los animales eran de las zonas de Azángaro, Puno (3827 msnm) y de Sama, Tacna (175 msnm). Se empleó un electrocardiógrafo Dong Jiang ${ }^{\circledR} 11$ A, monocanal y pinzas eléctricas adaptadas para uso electrocardiográfico veterinario. 
El análisis de los registros electrocardiográficos se realizó en el Laboratorio de Patología Clínica de la Facultad de Medicina Veterinaria y Zootecnia (FMVZ) de la Universidad Nacional del Altiplano (UNAP), Puno, Perú. Se determinó la frecuencia, ritmo, tamaño y morfología de las ondas y el eje eléctrico cardíaco.

La investigación contempló los principios y directrices internacionales para la investigación biomédica y fue autorizada por el Comité de Ética y Bienestar Animal de la FMVZ-UNAP.

Los animales fueron sujetados manualmente y mantenidos en posición erguida por cerca de 20 min esperando que se tranquilicen. Se le colocaron las pinzas eléctricas en los pliegues axilares y en las babillas de los cuatro miembros. Para esto, las pinzas se humedecieron en el lugar de contacto con alcohol y se conectaron los terminales de los electrodos del electrocardiógrafo en cada pinza siguiendo el código internacional de colores. El electrocardiógrafo se mantuvo a una velocidad de $25 \mathrm{~mm} / \mathrm{seg}$ y a una sensibilidad de $1 \mathrm{mV} / 10 \mathrm{~mm}$. El registro se inició con las derivaciones DI, DII, DIII, avL, avR y avF, cada una por 6 seg (Pompa et al., 2001a).

Se consideró el promedio de las distancias R-R en la derivación II y III, en dos ciclos consecutivos, dividiendo entre 60 la distancia R-R (Montejo, 2006).

Se analizó el ritmo cardíaco normal descartando intervalos R-R asimétricos. Seguidamente se observó si a cada complejo QRS le antecedía la onda P. Se determinó la distancia entre el comienzo de la onda $\mathrm{P}$ y la onda Q (Montejo, 2006).

Se evaluaron los parámetros mensurables en duración, amplitud y morfología de las ondas electrocardiográficas. Se determinó la duración de las ondas P, QRS y $\mathrm{T}$, el valor de los intervalos P-R, QRS, R-R, Q-T y T-P, la amplitud de las ondas P, QRS y $\mathrm{T}$ y el valor de los segmentos PQ y ST (Montejo, 2006).
Se calculó la media y el desvío estándar de cada variable y se utilizó la prueba de «t» de Student para comparar muestras independientes para determinar posibles diferencias entre las medias del grupo de animales de altura con aquellos a nivel del mar (Martínez y Faulin, 2001). Se utilizó el paquete estadístico STATA 8.0.

\section{Resultados y Discusión}

\section{Frecuencia Cardíaca}

El Cuadro 1 presenta los valores del electrocardiograma referidos a frecuencia cardíaca e intervalo R-R. Todos los animales completaron el ciclo cardíaco (intervalo R$\mathrm{R})$. Las frecuencias cardíacas encontradas fueron concordantes con los valores reportados por Pérez (2002) a nivel de Sierra, en toretes y vaquillas con 80 a 90 latidos por minutos (lpm) y 65 a $77 \mathrm{lpm}$, respectivamente. Teniendo en cuenta que la frecuencia cardíaca es el parámetro más variable en el electrocardiograma, la literatura científica reporta una gran cantidad de trabajos (Pompa et al., 2001b) donde los diversos valores de los segmentos e intervalos dependen de este parámetro (Hilwig, 1982).

Los animales que viven en grandes alturas presentan una hipertrofia e hiperplasia cardiaca relacionada a la baja presión parcial de oxígeno atmosférico, produciendo un estrechamiento del lumen de arterias pulmonares; por otro lado, el diámetro transverso y longitudinal del corazón es más grande que en animales que viven a nivel del mar (Andrews, 2004). Estos cambios están relacionados con los procesos de adaptación fisiológica que sufren los animales al nacer y criarse en condiciones de altura, donde se encuentran sometidos a la disminución del oxígeno ambiental (hipoxia), de la presión atmosférica total y de la presión parcial del oxígeno disponible en el medio (Ayón y Cueva, 1998), exigiendo al organismo una serie de ajustes, expresados inicialmente en un aumento de las frecuencias cardíaca y respiratoria. 
Cuadro 1. Valores electrocardiográficos de la frecuencia cardíaca y el intervalo R-R en terneros Holstein criados en la altura (3827 msnm) y a nivel del mar (175 msnm)

\begin{tabular}{|c|c|c|c|}
\hline Nivel altitudinal & $\begin{array}{l}\text { Valores } \\
\text { electrocardiográficos }\end{array}$ & $\begin{array}{l}\text { Media } \pm \text { SD } \\
\quad(\text { Rango) }\end{array}$ & Morfología \\
\hline \multirow{2}{*}{ Altura } & $\mathrm{FC}(\mathrm{lpm})^{1}$ & $\begin{array}{l}87.3 \pm 9.8 \\
(62-125)\end{array}$ & Uniforme \\
\hline & R-R (seg) & $\begin{array}{c}0.34 \pm 0.05 \\
(0.18-0.48)\end{array}$ & $\begin{array}{l}\text { Simétrico, determina } \\
\text { los periodos cardíacos }\end{array}$ \\
\hline \multirow{2}{*}{ Nivel del mar } & FC (lpm) & $\begin{array}{c}127.40 \pm 7.52 \\
(115-150)\end{array}$ & Uniforme \\
\hline & R-R(Seg) & $\begin{array}{c}0.61 \pm 0.09 \\
(0.4-0.8)\end{array}$ & $\begin{array}{l}\text { Simétrico, determina } \\
\text { los periodos cardíacos }\end{array}$ \\
\hline
\end{tabular}

${ }^{1}$ Frecuencia cardíaca (latidos por minuto)

Cuadro 2. Valores del electrocardiograma respecto al ritmo cardíaco ${ }^{1}$ en terneros Holstein criados en la altura (3827 msnm) y a nivel del mar (175 msnm)

\begin{tabular}{lllll}
\hline Nivel altitudinal & $\begin{array}{l}\text { Frecuencia } \\
\text { cardíaca } \\
(\mathrm{lpm})\end{array}$ & Onda P & Complejo QRS & $\begin{array}{l}\text { Intervalo } \\
\text { R-R } \\
(\mathrm{seg})\end{array}$ \\
\hline \multirow{2}{*}{ Altura } & $87.3 \pm 9.8$ & $0.02 \pm 0.00 \mathrm{seg}$ & $0.04 \pm 0.01 \mathrm{seg}$ & $0.34 \pm 0.05$ \\
& $\begin{array}{l}\text { Aparentemente } \\
\text { normal }\end{array}$ & Visible & $\begin{array}{l}\text { Visible } \\
\text { predominancia QRS }\end{array}$ & Simétrico \\
& $127.4 \pm 7.5$ & $0.038 \pm 0.01 \mathrm{seg}$ & $0.05 \pm 0.01 \mathrm{seg}$ & $0.61 \pm 0.09$ \\
Nivel del mar & $0.19 \pm 0.04 \mathrm{mV}$ & $1.48 \pm 0.10 \mathrm{mV}$ & Visible \\
& $\begin{array}{l}\text { Aparentemente } \\
\text { normal }\end{array}$ & Visible & predominancia QRS & Simétrico \\
\hline
\end{tabular}

${ }^{1}$ Ritmo regular sinusal normal

Los resultados a la evaluación del intervalo RR muestran que el ciclo cardíaco es menor en la altura $(0.34 \mathrm{seg})$ que a nivel del mar $(0.61 \mathrm{seg})$. De este resultado se deduce que las diferencias en la presión atmosférica total y parcial de oxígeno y de temperatura ambiental (que suele ser más fría en la altura) podrían explicar las diferencias encontradas (Rezakhani et al., 2004). Además, los animales criados en la altura registran una mayor actividad del sistema hematógeno al estar sometidos a hipoxia celular, lo que estimula la eritropoyetina que induce la elevación de la hemoglobina sanguínea y, por ende, un aumento en el número de glóbulos rojos (Health y Reid, 1974; Kolb, 1975; Gurtler, 1976; Guyton y Hall, 2006). Por tanto, la disminución en la presión de oxígeno atmosférico reduce el transporte del oxígeno desde los pulmones hasta las mitocondrias celulares, lo 
Cuadro 3. Valores electrocardiográficos de ondas, segmentos e intervalos en terneros Holstein criados en la altura (3827 $\mathrm{msnm})$ y a nivel del mar (175 msnm)

\begin{tabular}{|c|c|c|c|c|}
\hline \multirow{2}{*}{$\begin{array}{l}\text { Valores } \\
\text { electrocardio- } \\
\text { gráficos }\end{array}$} & \multicolumn{2}{|c|}{ En la altura } & \multicolumn{2}{|c|}{ A nivel del mar } \\
\hline & Media \pm de & Morfología & Media \pm de & Morfología \\
\hline $\mathrm{P}(\mathrm{seg})$ & $0.02 \pm 0.001$ & Picuda de aspecto & $0.04 \pm 0.01$ & Redondeada de \\
\hline $\mathrm{P}(\mathrm{mV})$ & $0.20 \pm 0.023$ & normal & $0.19 \pm 0.04$ & aspecto normal \\
\hline QRS (seg) & $0.04 \pm 0.006$ & Predominancia & $0.05 \pm 0.008$ & Predominancia \\
\hline QRS (mV) & $1.18 \pm 0.397$ & QRS & $1.48 \pm 0.10$ & QRS \\
\hline $\begin{array}{l}\mathrm{T}(\mathrm{seg}) \\
\mathrm{T}(\mathrm{mV})\end{array}$ & $\begin{array}{l}0.03 \pm 0.008 \\
0.23 \pm 0.084\end{array}$ & $\begin{array}{l}\text { Redondeada, más alta } \\
\text { que la onda P y } \\
\text { puntiaguda en la } \\
\text { parte superior }\end{array}$ & $\begin{array}{l}0.06 \pm 0.018 \\
0.38 \pm 0.082\end{array}$ & $\begin{array}{l}\text { Redondeada, más } \\
\text { puntiaguda en la } \\
\text { parte superior }\end{array}$ \\
\hline PR (seg) & $0.07 \pm 0.01$ & $\begin{array}{l}\text { Comprende desde la } \\
\text { onda P y una línea } \\
\text { isoeléctrica antes del } \\
\text { complejo QRS }\end{array}$ & $0.13 \pm 0.02$ & $\begin{array}{l}\text { Comprende desde } \\
\text { la onda P y una } \\
\text { línea isoeléctrica } \\
\text { antes del complejo } \\
\text { QRS }\end{array}$ \\
\hline QT (Seg) & $0.15 \pm 0.03$ & $\begin{array}{l}\text { Comprende al } \\
\text { complejo QRS y la } \\
\text { onda T }\end{array}$ & $0.18 \pm 0.03$ & $\begin{array}{l}\text { Comprende al } \\
\text { complejo QRS y la } \\
\text { onda T }\end{array}$ \\
\hline TP (seg) & $0.15 \pm 0.04$ & Línea isoeléctrica & $0.13 \pm 0.02$ & Línea isoeléctrica \\
\hline PQ (seg) & $0.04 \pm 0.01$ & Línea isoeléctrica & $0.016 \pm 0.003$ & Línea isoeléctrica \\
\hline ST (seg) & $0.14 \pm 0.03$ & Línea isoeléctrica & $0.10 \pm 0.01$ & Línea isoeléctrica \\
\hline
\end{tabular}

Cuadro 4. Determinación del eje electrocardiográfico mediante las derivaciones I, III $^{1}$ y su respectiva comprobación con las tablas de Tilley en terneros Holstein criados en la altura (3827 $\mathrm{msnm})$ y a nivel del mar (175 msnm)

\begin{tabular}{|c|c|c|c|c|c|c|c|}
\hline \multirow{2}{*}{$\begin{array}{l}\text { Nivel } \\
\text { altitudinal }\end{array}$} & \multicolumn{3}{|c|}{ Derivación I } & \multicolumn{3}{|c|}{ Derivación III } & \multirow{2}{*}{$\begin{array}{l}\text { Tablas } \\
\text { Tilley }\end{array}$} \\
\hline & Q & $\mathrm{R}$ & $\begin{array}{c}\text { Suma } \\
\text { algebraica }\end{array}$ & Q & $\mathrm{R}$ & $\begin{array}{c}\text { Suma } \\
\text { algebraica }\end{array}$ & \\
\hline $\begin{array}{l}\text { En la } \\
\text { altura }\end{array}$ & $\begin{array}{c}-4.3 \\
(1.84)\end{array}$ & $\begin{array}{c}2.5 \\
(2.29)\end{array}$ & $\begin{array}{c}0.1 \\
(3.25)\end{array}$ & $\begin{array}{c}-3.5 \\
(1.08)\end{array}$ & $\begin{array}{c}3.4 \\
(1.61)\end{array}$ & $\begin{array}{c}0.2 \\
(2.26)\end{array}$ & $\begin{array}{l}-120.1^{\mathrm{a}} \\
(7.30)\end{array}$ \\
\hline $\begin{array}{l}\text { A nivel } \\
\text { del mar }\end{array}$ & $\begin{array}{c}-2.4 \\
(0.32=\end{array}$ & $\begin{array}{c}5.8 \\
(0.64)\end{array}$ & $\begin{array}{c}3.3 \\
(0.58)\end{array}$ & $\begin{array}{l}-3.6 \\
(0.52)\end{array}$ & $\begin{array}{c}7.9 \\
(0.79)\end{array}$ & $\begin{array}{c}4.5 \\
(1.064)\end{array}$ & $\begin{array}{c}63.7^{\mathrm{b}} \\
(3.02)\end{array}$ \\
\hline
\end{tabular}

${ }^{1}$ Promedio (desviación estándar)

a,b Superíndices diferentes dentro de columnas indican diferencia estadística $(p<005)$ 
que implica que el animal recurra a esfuerzos propios para lograr la aclimatación a la nueva presión parcial de oxígeno (Arango et al., 1992).

Las diferencias observadas en la frecuencia cardíaca reflejan un balance más adecuado de las funciones simpáticas y parasimpáticas en los terneros, ya que estos se modifican con el pasar del tiempo (Álvarez et al., 1986). Es así que estos cambios han sido asociados a la época del año y a la edad de los animales (Rowlands et al., 1983; Siga et al., 1985). Se conoce que los animales en el invierno aumentan su producción de hormonas tiroideas, lo que se traduce en cambios asociados al gasto cardíaco para fines de mantenimiento de la temperatura y aumento de la producción energética, $\mathrm{y}$, asimismo, asociado a una disminución relativa de las pasturas (Ganong, 2010).

\section{Ritmo Cardíaco}

El ritmo cardíaco en la altura y a nivel del mar fue regular sinusal normal (Cuadro 2). No hubo diferencias estadísticas entre grupos debido a que el número de complejos R-R fue simétrico, la aparición de complejos QRS fue regular y hubo relación entre la onda P y el complejo QRS, lo que indica que el impulso salió del nódulo Keith-Flack (Montoya e Ynaraja, 2007). Otros autores sostienen que el ritmo cardíaco no es patológico si no es sinusal (Montejo, 2006).

\section{Ondas, Segmentos e Intervalos}

En el Cuadro 3 se muestran los valores obtenidos en ondas, segmentos e intervalos, donde en el caso de terneros en la altura, la duración y amplitud de la despolarización auricular (onda p) fue de $0.02 \pm 0.001 \mathrm{seg}$ y de $0.20 \pm 0.023 \mathrm{mV}$, respectivamente, en tanto que en los terneros criados a nivel del mar fue de $0.04 \pm 0.01 \mathrm{seg}$ y $0.19 \pm 0.05 \mathrm{mV}$, respectivamente. Estos resultados indican que los terneros en la altura presentan un ventrículo ligeramente agrandado (por las características que presenta la onda $\mathrm{P}$ que es alta y termina en punta más parecida o semejante a una $\mathrm{P}$ pulmonar), relacionado directamente con la hipoxia y la acción de las ramas simpáticas de secreción noradrenérgica y parasimpáticas de secreción colinérgica que están presentes en el complejo auricular y son nulas en los ventrículos (Cunninham, 2003).

En relación a tiempo de conducción aurículo-ventricular en la altura, el intervalo PR fue de $0.07 \pm 0.01 \mathrm{seg}$ y el intervalo PQ fue de $0.04 \pm 0.01 \mathrm{seg}$, valores menores a los encontrados a nivel del mar $(0.13 \pm 0.02 \mathrm{seg}$ y $0.016 \pm 0.003 \mathrm{seg}$, respectivamente, Cuadro 2). Estos datos expresan un retraso en el paso del impulso eléctrico a nivel aurículoventricular, donde el acortamiento no es considerado patológico sino como un exceso de tono vagal estando en reposo, produciéndose una cantidad moderada de descarga tónica en los nervios simpáticos. Esto, en general es característico en humanos y en animales grandes (Ganong, 2010).

En los procesos de contracción, despolarización y contracción de los ventrículos (complejo QRS) en la altura se observaron valores $\mathrm{QRS}$ promedio de $0.04 \pm$ 0.006 seg y de $1.18 \pm 0.397 \mathrm{mV}$, mientras que a nivel del mar se obtuvieron valores de $051 \pm 0.008 \mathrm{seg}$ y $1.48 \pm 0.10 \mathrm{mV}$. El aumento de la amplitud del complejo QRS se produce por un aumento del tono simpático, que depende del tamaño del corazón y también de las modificaciones que se experimentan en las vías de conducción eléctrica cardíaca de los animales bajo determinadas condiciones del estrés animal (Pompa, 2000).

La deflexión negativa que produce la despolarización de la parte apical del septo interventricular medial (onda Q) en terneros en altura fue de $-1.09 \pm 0.56 \mathrm{mV}$ y a nivel del mar fue de $-0.38 \pm 0.028 \mathrm{mV}$. La mayor amplitud de la onda $Q$ en terneros en altura se debe a que la corriente eléctrica pasa por un músculo hipertrofiado, el cual tiene que ven- 
cer la mayor resistencia vascular a nivel pulmonar (Kittleson y Kienle, 2000), ante una esperada sobrecarga de presión o volumen en el ventrículo derecho (Cuninham, 2003).

La segunda onda negativa que representa la despolarización de las zonas altas de las paredes ventriculares y del septo ventricular (onda $S$ ) fue de $-0.06 \pm 0.03 \mathrm{mV}$ en terneros en altura y de $-0.3 \pm 0.13 \mathrm{mV}$ a nivel del mar. La onda $\mathrm{S}$ en la altura no fue tan predominante como a nivel del mar, ello teniendo en cuenta que la onda S sirve también para determinar si hay hipertrofia cardíaca u otras variaciones en el lado derecho (San Román, 2000; Montoya e Ynaraja, 2007).

El periodo de repolarización y llenado ventricular (onda $\mathrm{T}$ ) tuvo una duración y amplitud en los terneros de altura de $0.03 \pm$ $0.008 \mathrm{seg}$ y $0.23 \pm 0.084 \mathrm{mV}$, en tanto que fue de $0.06 \pm 0.018$ seg y $0.38 \pm 0.082 \mathrm{mV}$ a nivel del mar (Cuadro 3). En la altura, las ondas tendieron a ser más delgadas y puntiagudas. Se debe tener en cuenta que la onda T es la que más se asocia a patologías, y cuando disminuye también lo hace la frecuencia cardíaca. Esta onda, generalmente es más puntiaguda en caso de que los animales presenten hipoxia por ventilación deficiente, por lo que cualquier patología respiratoria que curse con una ventilación deficiente produce alteraciones en esta onda (Montoya e Ynaraja, 2007).

Las diferencias encontradas en la fase de repolarización ventricular (segmento S-T) entre terneros en altura $(0.14 \pm 0.03 \mathrm{seg})$ y a nivel del mar $(0.10 \pm 0.01 \mathrm{seg}$; Cuadro 3$)$ están relacionadas con hipertrofia ventricular (Santamarina et al., 2001), aunque también puede ser secundaria a cambios o anormalidades en el complejo QRS (San Román, 2000).

En relación a la recuperación ventricular (intervalo QT), DeRoth (1980) sostiene que los terneros al nacimiento tienen una mayor ampliación de la zona cardíaca, siendo la dis- minución de la frecuencia cardíaca una característica que se produce naturalmente durante el crecimiento de los animales.

Los datos obtenidos durante el reposo del músculo cardíaco (intervalo TP) fueron similares en ambos grupos (Cuadro 3). Se encontró diferencia significativa entre el tamaño y la morfología de las ondas, segmentos e intervalos en la altura y a nivel del mar $(\mathrm{p}<0.05)$.

\section{Eje Electrocardiográfico}

Los valores del trazado del eje electrocardiográfico fueron de $-120.1 \pm 7.30^{\circ}$ en terneros en altura y de $63.7 \pm 3.02^{\circ}$ a nivel del mar ( $<<0.05$; Cuadro 4), lo cual demuestra que en los primeros el vértice del corazón tiene una dirección hacia el extremo derecho, característica que se presenta generalmente cuando un ventrículo sufre elevada hipertrofia, por lo que el eje del corazón se desvía hacia el ventrículo hipertrófico. Esto ocurre debido a que el grosor muscular en el lado hipertrofiado es mayor en comparación del otro, y que la onda de despolarización tarda más tiempo en pasar por el ventrículo hipertrófico que si atraviesa un corazón normal (Ghina et al., 2008a,b).

Las variaciones se justifican porque la actividad eléctrica debe ser significativa para llegar al vértice del corazón durante la activación del complejo QRS (Amory et al., 1993), pero también se asocian a las características anatómicas y fisiológicas del corazón del bovino (Ghiñã et al., 2008a,b). El complejo de Purkinje esté más desarrollado en animales mayores en relación a animales jóvenes (Cunningham, 2003). Independientemente de la altitud, las diferencias en cuanto a las demandas energéticas y metabólicas parecen influir en las ondas electrocardiográficas en diferentes porciones. Las variaciones de la electroconductividad de los tejidos están asociadas a modificaciones en el estado fisiológico de los animales, los cuales influyen en actividad del miocardio (Pompa et al., 2001a). 


\section{Conclusiones}

- Los parámetros electrocardiográficos en altura y a nivel del mar en terneros Holstein muestran diferencias significativas $(\mathrm{p}<0.05)$ para frecuencia cardíaca y tamaño de las ondas.

- El ritmo cardíaco se caracterizó por un ritmo regular sinusal normal.

- La morfología de las ondas en los animales de altura tuvo predominancia aguzada.

- El eje electrocardiográfico en la altura se caracterizó por un eje ubicado extremamente a la derecha, a diferencia de los animales a nivel del mar cuyo eje está dirigido al lado izquierdo, lo cual se considera normal para la especie.

\section{Literatura Citada}

1. Alvarez J, Zaldívar V, Colomé H, Mayarí R. 1986. Indicadores hematoquímicos de toros sementales para inseminación artificial. I. Efecto de la época del año. Rev Salud Anim 8: 287-294.

2. Amory H, Genicot B, Rollin F. 1993. Normal electro- and vectocardiographic data in the growing Friesian calf using a semi-orthogonal lead system. J Vet Med 40: 81-92. doi: 10.1111/j.1439-0442. 1993.tb00604.x

3. Andrews A. 2004. Bovine medicine: diseases and husbandry of cattle. Oxford, UK: Blackwell Sciencia Publishing. 1131 p.

4. Arango C, Oquendo R, Agudelo G. 1992. Influencia de la altitud en parámetros fisiológicos generales y hemáticos de bovinos Holstein. Rev Fac Nal Agr Medellín: 45: 51-60.

5. Ayón M, Cueva S. 1998. Adaptación del ganado bovino a la altura. Pub. Téc. $\mathrm{N}^{\mathrm{o}}$ 38. Facultad de Medicina Veterinaria, Univ. Nacional Mayor de San Marcos. Lima. 15 p.
6. Claxton M. 1988. Electrocardiographic evaluation of arrhythmias in six cattle. $J$ Am Vet Med Assoc 192: 516-521.

7. Cunningham J. 2003. Fisiología veterinaria. $2^{\mathrm{a}}$ ed. México DF: McGraw-Hill Interamericana. $775 \mathrm{p}$.

8. DeRoth L. 1980. Electrocardiographic parameters in the normal lactating Holstein cow. Can Vet J 21: 271-277.

9. [FAO] Organización de las Naciones Unidas para la Alimentación y la Agricultura. 2008. Ayudando a desarrollar una ganadería sustentable en América Latina y el Caribe: lecciones a partir de casos exitosos. Santiago, Chile. [Internet]. Disponible en: http:/ www.fao.org/docrep/010/i0082s/ i0082s00.HTM

10. Fregin F. 1985. Electrocardiography. Vet Clin North Am Equine Pract 1: 419432.

11. Ganong $W .2010$. Fisiología médica. México: Interamericana-McGraw Hill. $720 \mathrm{p}$.

12. Ghiñã M, Cotor G, Brãslasu C. $2008 a$. The values of ECG parameters in newborn calves. Bull UASVM 65: 473

13. Ghiñã M, Cotor G, Brãslasu, C. $2008 \mathrm{~b}$. The values of some ECG components in dairy cows, using Dubois leads. Bull UASVM 65: 474.

14. Guyton A, Hall J. 2006. Tratado de fisiología médica. España: Elsevier. 1104 p.

15. Gurtler H. 1976. Fisiología veterinaria. Zaragoza: Acribia. 569 p.

16. Heatlth D, Reid D. 1974. Life height altitude. Edwaard Arnold. 60 p.

17. Hilwig $R \boldsymbol{W}$. 1982. ECG of the month. J Am Vet Med Assoc 181: 128-129.

18. Kittleson M, Kienle R. 2000. Medicina cardiovascular de pequeños animales. $2^{\mathrm{a}}$ ed. Barcelona: Ed Multimédica. 603 p.

19. Kolb E. 1975. Corazón y circulación. In: Gurtler H, Ketz HA, Kolb E, et al. (eds). Fisiología veterinaria. Zaragoza: Acribia. p 560-610.

20. Martínez H, Faulin F. 2001. Bioestadística amigable. Madrid: Ed Díaz Santos. 520 p. 
21. Montejo E. 2006. Electrocardiografía veterinaria y enfermedades cardiovasculares. Ecuador: Ed Universidad de Cuenca. $126 \mathrm{p}$.

22. Montoya J, Ynaraja E. 2007. Manual práctico de electrocardiografía en pequeños animales. España: Elsevier-Masson. $241 \mathrm{p}$.

23. Pérez G 2002. Diagnóstico físico en vacunos. Puno, Perú: Ed Universitaria UNA. $188 \mathrm{p}$.

24. Pompa A, Marrero E, Fernández O, González R. 2001a. Comportamiento del electrocardiograma en bovinos de la raza Holstein a diferentes edades de uno y otro sexo. Rev Salud Anim 23: 49-55.

25. Pompa A, Marrero E, Fernández O, González R. 2001b. Influencia de las estaciones del año sobre el electrocardiograma en bovinos de la raza Holstein. Rev Salud Anim 23: 42-48.

26. Pompa A. 2000. Nomenclatura del complejo QRS en animales del grupo B. Rev Salud Anim 22: 35-40.

27. Rezakhani A, Paphan A, Shekarfroush S. 2004. Analysis of base apex lead electrocardiograms of normal dairy cows. Vet Arch 74: 351-358.
28. Rowlands G, Mauston R, Kathrym J, Brookes P. 1983. A genetic analysis of the concentration of blood metabolites and their relationships with age and live weight gain in young British Friesian Bulls. Livestock Prod Sci 10: 1-16. doi: 10.1016/0301-6226(83)90002-7

29. San Roman F. 2000. Canis et Felis: interpretación de electrocardiogramas. $5^{a}$ ed. México: Luzán. 101 p.

30. Santamarina G, Espino L, Suarez $L$. 2001. Electrocardiographic parameters of free-ranging roe deer (Capreolus capreolus). J Zoo Wildl Med 32: 441446.

31. Siga A, Abe K, Kamemoto S, Keino M, Tsukamoto K, Fujio O. 1985. Effects of age, milking and season on magnesium, calcium and inorganic phosphorus metabolisms in cows. Nihon Juigaku Zasshi 47: 275-283.

32. Tilley L, Lee N. 2000. Electrocardiografía práctica en pequeños animales. Manual de consulta rápida. España: Multimédica. $108 \mathrm{p}$.

33. West J. 2003. Effects of heat-stress on production in dairy cattle. J Dairy Sci 86: 2131-2144. doi: 10.3168/jds.S00220302(03)73803-X 